\section{AB0397 PREVALENCE AND FACTORS ASSOCIATED WITH DEPRESSION AMONG PATIENTS AFFECTED BY CHRONIC INFLAMMATORY ARTHRITIS: PRELIMINARY RESULTS OF A SINGLE-CENTRE EXPERIENCE IN ITALY}

S. Pezzato ${ }^{1}$, S. Tomassi ${ }^{2}$, E. Fracassi ${ }^{1}$, A. Giollo ${ }^{1}$, C. Caimmi ${ }^{1}$, F. Martinis ${ }^{1}$, M. G. Gnatta ${ }^{2}$, D. Cristofalo ${ }^{2}$, C. Bonetto ${ }^{2}$, M.L. Marognolli ${ }^{1}$, S. Tosato ${ }^{2}$, A. Carletto ${ }^{1}$. ${ }^{1}$ Rheumatology, Reumatologia - AOUI Verona; ${ }^{2}$ Section of Psychiatry, Department of Neurosciences, Biomedicine and Movement Sciences, University of Verona, Verona, Italy

Background: Depression and anxiety disorders are more common in chronic inflammatory arthritides (CIA) than in the general population. ${ }^{1}$ In rheumatoid arthritis (RA), depressive and anxiety symptoms have been associated with disease activity and pain perception, ${ }^{1}$ physical disability, ${ }^{2}$ health care costs, ${ }^{3}$ and mortality. ${ }^{4}$ To our knowledge, data on the prevalence of depression in Italian patients with RA are scarce.

Objectives: To estimate the prevalence and factors associated with depression among patients affected by RA.

Methods: During an one year period, patients affected by RA have been consec utively enrolled. For each patient demographics and disease characteristics were recorded; the Hospital Anxiety and Depression Scale (HADS) was administered. HADS is a validate questionnaire to assess depressive and anxiety symptoms in patients affected by somatic disorders admitted to general hospital. An HADS $\geq 11$ was considered diagnostic for depression.

Results: The table summarises the main results. We recruited 231 patients affected by RA, finding a prevalence of depression of $13.9 \%(95 \% \mathrm{Cl} 9.4 \%-$ $18.3 \%)$. Overall, patients with depression, when compared with patients without, were older, more frequently females, with a lower education, higher physician global assessment and patient global assessment, more comorbidities. In particular, they had any cardiovascular, neurologic or musculoskeletal issue $(p=0.018$, $p=0.032, p=0.014$, respectively) and more often used glucocorticoids or NSAIDs. Conversely, no association was found between depressive symptoms and the use of biologic therapies or the presence of inflammatory markers. Interestingly, a positive history of depression was present only in few patients with an HADS $>11$.

Abstract AB0397 - Table 1

\begin{tabular}{lccc}
\hline & $\begin{array}{c}\text { Non-depressed } \\
(\mathbf{n}=\mathbf{1 9 9})\end{array}$ & $\begin{array}{c}\text { Depressed } \\
(\mathbf{n}=\mathbf{3 2})\end{array}$ & P value \\
\hline Age, years & $57.5(12.3)$ & $63.5(11)$ & 0.008 \\
Female sex & $152(76)$ & $29(91)$ & 0.049 \\
Employed & $86(43)$ & $5(16)$ & 0.002 \\
Higher education & $89(45)$ & $8(25)$ & 0.036 \\
Disease duration, years & $12(9.2)$ & $14.2(8.3)$ & 0.202 \\
Erosive disease & $72(36)$ & $12(38)$ & 0.516 \\
TJC 0-28 & $1.9(3.4)$ & $2.3(2.6)$ & 0.500 \\
SJC 0-28 & $0.9(2.2)$ & $1.1(2.0)$ & 0.688 \\
PhGA 0-10 & $3.9(2.7)$ & $5.6(2.4)$ & 0.001 \\
PGA 0-10 & $4.9(2.4)$ & $6.8(2.0)$ & $<0.001$ \\
ESR, mm/h & $19.2(16.1)$ & $22.2(13.5)$ & 0.326 \\
CRP, mg/L & $6.4(14)$ & $4.4(3.8)$ & 0.425 \\
Comorbidities & $176(88)$ & $32(100)$ & 0.027 \\
CSDMARD & $136(68)$ & $18(56)$ & 0.127 \\
bDMARD & $129(65)$ & $24(75)$ & 0.177 \\
Glucocorticoid & $87(44)$ & $20(63)$ & 0.037 \\
NSAID & $32(16)$ & $12(38)$ & 0.007 \\
Antidepressant & $18(9)$ & $11(34)$ & $<0.001$ \\
\hline
\end{tabular}

Conclusions: These preliminary results suggest that more than one every seven patients with RA could suffer from depression, thus depression might be undiagnosed in a significant number of patients. Comorbidities, anti-inflammatory medications and both physician- and patient- driven evaluation of disease burden were the most striking factors associated with depression in this cohort of patients with RA.

\section{REFERENCES}

[1] Kojima M, et al. Arthritis and rheumatism 2009 Aug 15;61(8):1018-24.

[2] Peck JR, et al. Arthritis and rheumatism 1989 Sep;32(9):1100-6.

[3] Joyce AT, et al. J Rheumatol 2009 Apr;36(4):743-52.

[4] Ang DC, et al. J Rheumatol 2005 Jun;32(6):1013-9.

Disclosure of Interest: None declared

DOI: 10.1136/annrheumdis-2018-eular.5152

\section{$\mathrm{AB0398}$ \\ INVESTIGATION OF PREOPERATIVE INTRANASAL COLONISATION IN ORTHOPAEDIC SURGERY FOR PATIENT WITH RHEUMATOID ARTHRITIS}

S. Tsuji ${ }^{1}$, J. Hashimoto ${ }^{1}$, T. Noguchi ${ }^{2}$, S. Akita ${ }^{2}$, K. Yachi $^{2}$, M. Saito ${ }^{2}$, S. Ohshima ${ }^{1}$, Y. Saeki ${ }^{3}$. ${ }^{1}$ Department of RheumatologyandAllergology; ${ }^{2}$ Department of Orthopaedic Surgery; ${ }^{3}$ Department of Clinical Research, National Hospital Organization, Osaka Minami Medical Center, Kawachinagano City, Osaka, Japan

Objectives: Coagulase-negative staphylococci (CoNS) is listed as the major SS causative organism in the field of orthopaedic surgery, but detailed report on the presence or absence of methicillin-resistant CoNS of intranasal colonisation is not found.

Methods: In 13 months from July 2016 to the end of July 2017, 1000 cases of consecutive cases in which nasal cavity culture was performed within one month prior to hospitalisation for patients scheduled for our orthopaedic surgery. 223 men, 777 women, average age 66.4 years old, 197 cases of RA patients, 803 cases except RA patients.

The method of intranasal culture is as follows. After culturing for 18 hours at $35^{\circ}$ C using blood agar medium (daily water) and CHROMager MRSA selective medium (Kanto Kagaku), identification of bacterial species of the genus Staphylococcus grown and cultivation of drug susceptibility tests It was carried out by BD Phoenix according to the method of the Association (CLSI).

Results: The results of intranasal culture showed that $\mathrm{S}$. aureus, S. epidermidis, CoNS (excluding S. Epidermidis) and culture negative were $18.2 \%, 27.8 \%, 7.8 \%$, and $23.9 \%$, respectively. MRSA, MRSE and MRCoNS (excluding MRSE) with methicillin resistance were $3.2 \%, 22.1 \%$ and $1.7 \%$, respectively, and $27.0 \%$ of methicillin resistant bacteria were found to exist.

Especially in patients with RA, the methicillin-resistant bacterial colonisation rate was significantly higher $(p<0.05)$ than $38.6 \%$ compared with $24.2 \%$ other than RA patients.

Conclusions: Methicillin-resistant Staphylococcus was found to be $27.0 \%$ intranasal colonisation of patients in orthopaedic surgery. Especially in RA patient, it was high rate of $38.6 \%$. In the future, we need to consider the selection of perioperative preventive antibacterial drugs.

Disclosure of Interest: None declared

DOI: 10.1136/annrheumdis-2018-eular.4890

\section{AB0399 1 AUDIT OF INFLUENZA AND PNEUMOCOCCAL VACCINATIONS IN A COHORT OF RHEUMATOID ARTHRITIS PATIENTS ON BIOLOGICS}

S. Selvan. Rheumatology, Basildon and Thurrock NHS Foundation Trust, Essex, UK

Background: Rheumatoid arthritis (RA) patients are at increased risk of pneumococcal and influenza disease secondary to a deficient immune system and immunosuppressive medications. ${ }^{1}$ Despite the possible reduction in vaccine efficacy in RA, prophylactic vaccination for preventing pneumococcal pneumonia and influenza in RA patients should be routinely advised and practised. ${ }^{2}$ EULAR guidelines recommend that influenza and pneumoccocal vaccinations should be strongly considered for all patients with $\mathrm{RA}^{3}$

Objectives: To study pneumococcal and influenza vaccination rates in a cohort of RA patients on biologic medications.

Methods: We undertook a prospective audit of RA patients on biologics attending routine rheumatology clinics in a DGH over a 6 week period. A proforma was given to patients, and data was collected on medications, comorbidities, Disease Activity Score (DAS) and vaccination status for influenza and pneumoccocus.

Results: Of the 25 patients audited, 17 (68\%) were female and $8(32 \%)$ were male. The average age of patients included was 59 years (range 37-75). 18 (72\%) patients were seropositive, and 7 (28\%) were seronegative. The patients included had the following comorbidities; 14 (56\%) none, $6(24 \%)$ thyroid disease $1(4 \%)$ osteoporosis, $1(4 \%)$ hypertension, 1 (4\%) type 2 diabetes, $1(4 \%)$ interstitial lung disease.

On reviewing their medications, $7(28 \%)$ patients were on an Etanercept biosimilar (Benpali), 2 (8\%) on Tociliumab, 2 (8\%) on Rituximab, 9 (36\%) on Adalimumab, 2 $(8 \%)$ on an Infliximab biosimilar (Remsima), 2 (8\%) on Etanercept, and 1 (4\%) on Certolizumab. In addition to their biologic agent, all 25 patients were on Methotrexate. 2 patients $(8 \%)$ were also on Sulphasalazine, and $2(8 \%)$ were on Hydroxychloroquine additionally. The average DAS CRP score was 2.7 .

$8(32 \%)$ patients had received both influenza and pneumococcal vaccinations, and $9(36 \%)$ patients had neither. On analysing the vaccination rates individually, $16(64 \%)$ patients were up to date with influenza and $7(28 \%)$ were up to date with pneumococcal. Of the 9 patients that had neither vaccination, $5(20 \%)$ patients were unaware of their requirement, and $3(12 \%)$ felt they were too unwell to receive them. $1(4 \%)$ patient was unsure whether they could have the vaccinations as they had been recruited into a research trial. 
Conclusions: Only $32 \%$ patients had received both influenza and pneumoccocal vaccinations. The uptake rate for influenza vaccination was reasonable at $64 \%$, however the rate for pneumococcal vaccination was only $28 \%$.

Vaccination assessment should be performed routinely for all RA patients in clinic, and in particular those on biologic medications to reduce the risk of developing these infections in this high risk cohort.

\section{REFERENCES:}

[1] Blumentals $W$, et al. Rheumatoid arthritis and the incidence of influenza and influenza-related complications: a retrospective cohort study. BMC Musculoskeletal Disorders 2012;13:158.

[2] Malemud C. Pneumonia and influenza vaccination in rheumatoid arthritis patients. Glob Vaccines Immunol 2016;1(3):60-62

[3] van Assen $S$, et al. EULAR recommendations for vaccination in adult patients with autoimmune inflammatory rheumatic diseases. Ann Rheum Dis $2011 ; 70: 414-422$.

Disclosure of Interest: None declared DOI: 10.1136/annrheumdis-2018-eular.2851

\section{AB0400 HIGH PREVALENCE OF COMORBIDITIES IN PATIENTS WITH RHEUMATOID ARTHRITIS IN SOUTH AFRICA}

S. Botha-Scheepers, A.G.A. Mohammed, A. Gcelu, B. Hodkinson. Medicine/ Rheumatology, Groote Schuur Hospital/University of Cape Town, Cape Town, South Africa

Background: Patients with rheumatoid arthritis (RA) are at increased risk to develop comorbidities. Data on the prevalence of comorbidities in RA patients in South-Africa is lacking. ${ }^{1}$ Poorly controlled joint inflammation, common use of glucocosteroids and nonsteroidal anti-inflammatory drugs, a high prevalence of smoking and obesity, together with a high burden of infectious diseases may be important risk factors for comorbidities in this population.

Objectives: To assess the prevalence of comorbidities in RA patients in Cape Town, South-Africa and the association with disease activity and DMARD use using the METEOR (Measurement of Efficacy of Treatment in the 'Era of Outcome' in Rheumatology) database.

Methods: This is an ongoing cross-sectional study. Data from 109 RA patients from regular clinic visits at Groote Schuur Hospital in Cape Town, South Africa have been entered in the Meteor database (from December 2016). The Meteor database is a free online tool that was developed to improve the management of RA patients by helping rheumatologists to register, monitor and tightly control disease activity. The tool is currently widely used in other countries, but has it has not yet been described in any African countries. Information on the following parameters were reported: demographics, disease duration, disease activity (CDAI), current DMARDs used and comorbidities.

Results: The mean age (SD) was 57.6 (14.7) years, disease duration (SD) 14.1 (14.6) years, female gender $86.7 \%$, RF positive $85.1 \%$ and ACPA positive $80.4 \%$. The average (SD) CDAl was 13.1 (9.8) and $49.3 \%$ were smokers. Current DMARDs used was Methotrexate (72\%), Sulphasalazine (29\%), Chloroquine $(67 \%)$, Low dose corticosteroids (47\%), Leflunomide (11\%), Etanacept (1\%), but no other biological agents (0\%). At least one comorbidity was present in $69 \%$ of the patients, two in $40 \%$, three in $26 \%$, four in $13 \%$ and five in $2 \%$ of the patients. The most frequently observed comorbid diseases were hypertension (45.5\%), tuberculosis (TB) (11.1\%), Diabetes Mellitis Type 2 (10.9\%) and osteoarthritis $(10.9 \%)$. Other diseases included hypercholesterolemia $(7.1 \%)$, gastro-oesophageal reflux disease/peptic ulcer (6.1\%), COPD/emphysema (6.1\%), HIV (4.0\%), hypothyroidism $(4.0 \%)$, ischaemic heart disease $(3.0 \%)$, liver disease $(3.0 \%)$, DVT/pulmonary embolism (3.0\%), malignancies (3.0\%), asthma (2.0\%), discoid lupus erythematosus $(2.0 \%)$, interstitial lung disease $(1.0 \%)$, anaemia $(1.0 \%)$, rheumatic heart disease (1.0\%), cerebrovascular accident (1.0\%) and depression (1.0\%).

Conclusions: This study shows a high prevalence of comorbidities among indigent patients with RA in South Africa. In particular, hypertension, TB and osteoarthritis were very common. More patients will be included in this study in the next few months. Furthermore, we will assess the association between comorbidities, disease activity and DMARD use. The METEOR tool offers the unique opportunity to study daily practice care as well as research questions in real life setting in a South African clinic. This study will provide information that is necessary to address the burden of comorbidities in patients with RA in South Africa.

\section{REFERENCE:}

[1] Dougados M, Soubrier M, et al. Prevalence of comorbidities in rheumatoid arthritis and evaluation of their monitoring: results of an international, cross-sectional study (COMORA). Ann Rheum Dis 2014;73:62-8.
Disclosure of Interest: None declared

DOI: 10.1136/annrheumdis-2018-eular.6994

\section{AB0401 ECHO-CARDIOGRAPHIC ABNORMALITIES IN RHEUMATOID ARTHRITISPATIENTS COMPARED TO AGE AND SEX-MATCHED CONTROLS}

S. Hannawi ${ }^{1}$, K. Naeem ${ }^{2} .{ }^{1}$ Rheumatology, Ministry of Health and Prevention of UAE; ${ }^{2}$ Cardiology, Ministry of Health and Prevention of United Arab Emirates, Dubai, United Arab Emirates

Background: Rheumatoid arthritis (RA) is a chronic inflammatory autoimmune disease of the joints with several extra-articular features. Cardiovascular disease (CVD) mortality accounts for $40 \%-50 \%$ of all deaths in RA. Apart from atherosclerotic heart disease other cardiac abnormalities had been found to be prevalent in RA; including, pericarditis, heart failure, coronary vasculitis and valve disease. Objectives: Due to scarcity of data regarding cardiac disease in the Middle East population, we studied echocardiographic features in RA patients compared to their age, sex, and traditional CVD risk factors matched controls.

Methods: In a cross-sectional study, we recruited 39 RA patients meeting the 1987 revised criteria of RA and 37 age, sex and traditional CVD risk factors matched controls. Standard trans-thoracic echocardiography examination was carried out by a specialties cardio-sonographer who was blinded to the status of the participants. Left ventricular dimensions, wall geometry, ejection fraction, diastolic parameters, right ventricular size and function, valve structure and function, pericardium, pulmonary pressures and aortic root dimensions were assessed by echocardiography. t-test and chi-2 test were used to compare the echocardiographic findings between the two groups. $P$ value of $<0.05$ was considered significant.

Results: Thirty-nine RA patients (34 F, $4 \mathrm{M}$ ) and 37 controls (32 F, $5 \mathrm{M}$ ) were studied. Among RA, 27 (69\%) were RF positive. The two groups were similar in terms of age $(p=0.86)$, gender $(p=0.71)$, and traditional cardiovascular risk factors. No significant difference was found between RA and the controls in term of left ventricular ejection fraction, wall geometry, diastolic parameters, right ventricular size and function, valve disease, pulmonary pressures, pericardium and aortic root dimensions. However, left ventricular end-diastolic diameter $(43.11 \pm 1.14 \mathrm{vs}$. $39.35 \pm 0.84 \mathrm{~mm}$ respectively, $\mathrm{p}=0.01)$, end-systolic diameter $(24.39 \pm 0.70 \mathrm{~mm}$ vs $26.96 \pm 0.96$, respectively, $\mathrm{p}=0.03)$ and Left ventricular mass index $(79.83 \pm 5.11$ vs. $63.64 \pm 3.15$, respectively, $p=0.01$ ) were significantly higher in RA patients than in the controls.

Conclusions: Patients with rheumatoid arthritis have higher left ventricular enddiastolic and end-systolic dimensions, and greater left ventricular mass index compared to their age, sex and traditional CVD risk factors-matched controls. As the increase in the left ventricular mass index is a predictor of cardiac sudden death, echocardiography might be a simple non-invasive tool for cardiac risk screening in RA.

Disclosure of Interest: None declared

DOI: 10.1136/annrheumdis-2018-eular.5658

\section{AB0402 RECENT ONSET RHEUMATOID ARTHRITIS HAVE AND INCREASED LEFT ANTERIOR DESCENDING CORONARY ARTERY WALL THICKNESS: EVIDENCE OF SUBCLINICAL CORONARY ARTERY DISEASE}

S. Hannawi ${ }^{1}$, I. AI Salmi ${ }^{2}$, H. Hannawi ${ }^{3} .{ }^{1}$ Rheumatology, Ministry of Health and Prevention of UAE, Dubai, United Arab Emirates; ${ }^{2}$ The Royal Hospital, Muscat, Oman; ${ }^{3}$ Ministry of Health and Prevention of United Arab Emirates, Dubai, United Arab Emirates

Background: Rheumatoid arthritis (RA) is associated with increased cardiovascular disease (CVD). Using different methods, presence of sub-clinical atherosclerosis changes have been reported in RA. As no previous study has measured left anterior descending (LAD) coronary artery thickness in RA

Objectives: this study aimed to investigates atherosclerotic changes of coronary artery in early RA of 12 months of symptom onset.

Methods: 15 RA patients and 20 control subjects who matched 10 RA participants were included in the study. Demographic details, RA characteristics, and laboratory values of 15 RA patients at first presentation. None of the patients or the control participants had previous CVD/cerebrovascular events or diabetes and matched 10 of the RA group for age and sex. LAD coronary artery wall thick ness compared between RA and their matched control. Among early RA patients, Correlation was assessed between LAD coronary artery wall thickness and the demographic features, RA activity features, and cardiovascular risk factors.

Results: Within early RA, LAD coronary artery wall thickness was related to Disease Activity Score ( $p=0.025, \beta$-coefficient $0.066, \mathrm{Cl}: 0.010-0.122)$ and to the patient global assessment of disease activity at RA onset $(p=0.006, \beta$-coefficient $0.003, \mathrm{Cl}: 0.001-0.005)$ ), in a positive linear relationship. LAD wall thickness was 